\title{
Plasma S100A8/A9 heterodimer is an early prognostic marker of acute kidney injury associated with cardiac surgery
}

\author{
Zacharoula Nikolakopoulou*,1, Lauren R Hector ${ }^{1}$, Benedict C Creagh-Brown ${ }^{1}$, Timothy W \\ Evans $^{2}$, Gregory J Quinlan ${ }^{\ddagger, 1}$ \& Anne Burke-Gaffney ${ }^{*, \neq, 1}$ \\ ${ }^{1}$ Vascular Biology, Cardiovascular Sciences, National Heart \& Lung Institute Division, Faculty of Medicine, Imperial College London, \\ London, SW3 6LY, UK \\ ${ }^{2}$ National Institute for Health Research Respiratory Biomedical Research Unit, Royal Brompton \& Harefield NHS Foundation Trust, \\ London, SW3 6NP, UK \\ *Authors for correspondence: zac.nikolakopoulou@gmail.com \\ **Authors for correspondence: a.burke-gaffney@imperial.ac.uk \\ ${ }^{\ddagger}$ Authors contributed equally
}

\begin{abstract}
Aim: We investigated whether plasma levels of the inflammation marker S100A8/A9, could predict acute kidney injury (AKI) onset in patients undergoing cardiac surgery necessitating cardiopulmonary bypass (CPB). Patients \& methods: Plasma levels of S100A8/A9 and other neutrophil cytosolic proteins were measured in 39 patients pre- and immediately post-CPB. Results: All markers increased significantly post-CPB with S100A8/A9, S100A12 and myeloperoxidase levels significantly higher in patients who developed AKI within 7 days. S100A8/A9 had good prognostic utility for AKI, with an area under the receiver operating characteristic curve of 0.81 (95\% Cl: $0.676-0.949)$ and a cut-off value of $10.6 \mu \mathrm{g} / \mathrm{ml}(85.7 \%$ sensitivity and $75 \%$ specificity) irrespective of age. Conclusion: Plasma S100A8/A9 levels immediately after cardiac surgery, can predict onset of AKI, irrespective of age.
\end{abstract}

First draft submitted: 10 July 2018; Accepted for publication: 6 December 2018; Published online: 27 February 2019

Neutrophil activation occurs during cardiac surgery necessitating cardiopulmonary bypass (CPB) and contributes to the systemic inflammatory response syndrome that manifests in the majority of such patients, post operatively [1]. Systemic inflammatory response syndrome can precipitate organ failure and in particular, acute kidney injury (AKI) $[2,3]$. Previously, we showed in a retrospective analysis of nearly 1900 patients at the adult intensive care unit (AICU), Royal Brompton Hospital, London, UK - a study designed to establish the epidemiology of AKI after cardiac surgery - a $25.9 \%$ incidence of AKI classified according to AKI network (AKIN) criteria and a hospital mortality of $15.6 \%[2,3]$. We also previously showed in a small cohort of cardiac surgery patients that a novel plasma marker of endothelial dysfunction, Robo4, measured $2 \mathrm{~h}$ after surgery was higher in patients with AKI [4]. A link between neutrophil activation and cardiac surgery-associated (CSA) AKI has also been suggested [5]; but whether levels of neutrophil-derived plasma markers could likewise indicate onset of AKI, and at early time points, is not clear. Without a doubt, the need to predict and prevent AKI is one of the most pressing clinical problems to be addressed in patients undergoing cardiac surgery, especially in the elderly, in whom AKI is associated with considerable mortality [6]. There is generally an increasing need and interest in the identification of new predictive biomarkers of AKI at its early stages, which will enable the clinicians to identify the patients in danger and intervene in order to prevent further AKI development [7].

S100A8/A9 (also calprotectin or [MRP]8/14) is a leukocyte calgranulin protein that makes up $40 \%$ of the cytosolic protein content of neutrophils and is found at lower levels (5\%) in monocytes [8]. A low molecular weight, calcium and arachidonic acid-binding protein, S100A8/A9 is an alarmin with dual intracellular and extracellular functions [9]. S100A8/A9 plays a key role in the progression of inflammation [10], causing neutrophil migration to inflammatory sites [11]; induction of cytokine release from monocyte/macrophages [12] and also the potentiation of superoxide $\left(\mathrm{O}_{2}^{-}\right)$-generating NADPH oxidase activity [13]. Moreover, S100A8/A9 is also as highly sensitive marker for acute inflammation [14]. We have previously shown that plasma S100A8/A9 increased $2 \mathrm{~h}$ postsurgery 
in patients who have undergone cardiac surgery necessitating CPB [15]. Others have shown increased S100A8/A9 gene expression in circulating leukocytes following $\mathrm{CPB}$ [16].

S100A12 (EN-Rage) is another calgranulin protein expressed in human neutrophils, but to a lesser extent than S100A8/A9, and in monocytes but in lower levels [17]. S100A12 is also chemotactic for neutrophils and monocytes [18] but acts independently of S100A8/A9 [19]. Plasma S100A12 also increased after cardiac surgery in adults, with significantly higher levels in those patients requiring prolonged hospitalization of more than 7 days [20]. Moreover, raised levels of S100A12 in children undergoing cardiac surgery, corresponded to onset of acute lung injury [21].

Another neutrophil protein, MPO, has long been valued as a neutrophil activation marker in patients undergoing cardiac surgery with CPB [22,23]. Increased levels of plasma cell-free DNA (cfDNA), a key component of neutrophil extracellular traps (NETs), is also indicative of neutrophil activation. NETs are a novel antimicrobial mechanism formed from a meshwork of chromatin fibres (cfDNA) extruded from neutrophils and coated with antibacterial proteins [24]. Plasma levels of cfDNA were shown to be: a marker of mortality in intensive care unit (ICU) patients [25,26]; indicative of severity of injury in patients with polytrauma [27,28]; and higher in nonsurviving sepsis patients compared with survivors [29-31]. To our knowledge, peri-operative levels of cfDNA in patients undergoing CPB have not been investigated.

For a plasma marker to be a useful predictor of AKI, it will be necessary that levels are independent of age. Leukocyte function can change with age $[32,33]$. While some responses diminish with ageing; others are unaffected or exacerbated. In general, neutrophil properties, such as phagocytosis, microbiocidal function and chemotaxis decrease with age; whereas, the ability to mount a robust neutrophilia to infection is not altered [34,35]. In monocytes, production of some cytokines (IL-1 and IL-8) increases with age, while release of others (IL-6 and TNF) is decreased [35,36]. Effects of chronological age on S100A8/A9 release, at least in the context of acute systemic inflammation associated with $\mathrm{CPB}$ and cardiac surgery, has not been assessed.

Our exploratory study in a cohort of 39 patients investigates whether early neutrophil-derived plasma markers, particularly S100A8/A9, can serve as diagnostic indices of the likelihood of AKI onset, following cardiac surgery necessitating $\mathrm{CPB}$. In particular, given the risk of AKI in the elderly, which increases mortality rates and the increasing number of more elderly patients undergoing cardiac surgery, our aim was to identify a marker that would be useful, irrespective of patient age.

\section{Materials \& methods}

Patient population \& sample collection

Patients were recruited, sequentially, at the Royal Brompton Hospital (August 2009-December 2009) from those undergoing cardiac surgery necessitating $\mathrm{CPB}$. Indications were aortic or mitral valve repair or replacement (with or without additional cardiac procedures) and coronary artery bypass grafting (CABG). Recruitment was restricted to UK Caucasians, 16 years of age or over; without a congenital heart condition and excluding those who did not provide informed written consent. Blood was collected from an indwelling arterial cannula, pre-operatively (in the anesthetic room) and immediately post-CPB, for measurement of S100A8/A9, S100A12, MPO and cfDNA; also on post-operative day (POD) 1 and 2 for C-reactive protein (CRP), neutrophil numbers (PMNs) and white cell counts (WCC), and up to POD7 for creatinine measurements. Plasma was extracted conventionally and samples were stored at $-80^{\circ} \mathrm{C}$.

\section{S100 proteins \& inflammatory marker assays}

Commercially available sandwich ELISA kits were used to measure plasma levels of: S100A8/A9 ([S100A8/A9]2, BÜHLMANN Laboratories AG, Schönenbuch, Switzerland); S100A12 (Circulex, MBL International, MA, USA); and MPO (Boster Immunoleader, CA, USA). Plasma levels of cfDNA were quantified using an Invitrogen Quant$\mathrm{iT}^{\mathrm{TM}} \mathrm{dsDNA}$ high-sensitivity assay kit (Life Technologies, Paisley, UK). CRP and IL-8 were determined using the $\mathrm{N}$ high-sensitivity CRP monoassay with the BN ProsPec Nephelometer (Dade Behring, IL, USA) and a bead array assay (xMAP, Luminex, TX, USA), respectively.

\section{Clinical data}

Peri-operative data and variables were recorded, either manually, or using an automated clinical data collection system. Values for age, gender, BMI, creatinine and additive EUROscore (a widely employed clinical risk assessment tool); the nature of the procedure and duration of bypass and ischemic time were reported. Indicators of pulmonary 
dysfunction or respiratory failure: specifically, duration of mechanical ventilation, post-operative oxygenation, $\mathrm{PaO}_{2}: \mathrm{FiO}_{2}[\mathrm{PF}]$ ratio, first value recorded on return to ICU and the arteriolar-alveolar oxygen gradient were also calculated. Likewise, a severity of illness score, the 'acute physiology and chronic health evaluation' (APACHE II) score was computed. This is an integer ICU scoring system (zero to 71 ) with a score of 25 representing a $50 \%$ in hospital mortality rate. This score is calculated within the first $24 \mathrm{~h}$ of ICU admission and evaluates the severity of illness and organ failure. Creatinine levels, indicative of kidney injury, are one of the 12 physiological measurements used to calculate the score. ICU length of stay (LOS), level 3 care duration - a composite index reflecting the degree of physiological derangement and the requirements for supportive care - advanced therapies and monitoring at a level of 'intensive care' [37]; hospital LOS and mortality were also documented. Patients were defined as having AKI or not (non-AKI), using the AKIN classification system [2,3]. Stages of AKI are calculated as follows: an absolute increase of creatinine in plasma of $24.6 \mu \mathrm{g} / \mathrm{ml}$ from pre-operative levels compared with any measurement for 7 days postsurgery stage 1 ; two- to threefold for stage 2 and $>3$-fold for stage 3 .

The research was carried out in accordance with the Declaration of Helsinki (2013) of the World Medical Association and the study protocol was approved by the Research Ethics Committee for the Royal Brompton and Harefield NHS Foundation Trust. Informed, written consent was obtained (one day before surgery and after determining patient suitability) from all patients. Reporting of the study conforms to STROBE and broader EQUATOR guidelines [38].

\section{Statistics}

Statistical analyses were performed to establish how plasma biomarkers changed following surgery and to investigate associations with clinical indices. Nonparametric data were expressed as median and interquartile range (IQR) and compared using Wilcoxon-matched pairs test for two paired groups or Friedman's test for three or more paired groups, with Dunn's correction; Kruskal-Wallis with Dunn's post-test (for an unequal number of samples in three or more columns); or Mann-Whitney U test for two unpaired groups. Parametric data were expressed as mean \pm standard deviation (SD). Correlations between variables were assessed using Spearman's analysis (nonparametric, stating r) or Pearson's parametric analysis $\left(\mathrm{r}^{2}\right)$, with associated $\mathrm{p}$-values. P-values of $<0.05$ were considered significant. To measure sensitivity and specificity for plasma biomarkers and clinical indices, conventional receiver operating characteristic (ROC) curves were calculated. The area under the curve (AUC) was calculated to ascertain the utility of a molecule as a biomarker that can be used to distinguish between two groups, in other words, normal and diseased or lower versus higher risk, as in this study. An area of 0.5 is expected by chance, whereas an area of 1 signifies a perfect biomarker. Excel 2007 (Microsoft, CA, USA) and Prism v5.04 (Graphpad software, CA, USA) were used for analyses.

\section{Results}

\section{Patient characteristics}

Demographics of the patients ( $\mathrm{n}=39,66.7 \%$ male), clinical characteristics, operative and post-operative variables and outcomes are shown in Table 1. Mean age of 66.5 years was similar to the national average for patients undergoing CPB surgery [39]. Mean Euroscore of 4.87 indicates that patients did not have high pre-operative risk (i.e., Euroscore $<6)$. Ischemic time $(91.5 \pm 32.1 \mathrm{~min})$ was similar to mean aortic cross clamping time (85-87 min) reported in a recent study with patients of similar complexity [40]. Medium duration of level 3 care required (16.6 h) was comparable to that we have previously reported [15]. The incidence of AKI of $18 \%$ was similar to that published by us in a previous cohort study [4]. Increased WCC, PMNs, plasma CRP and IL-8 levels (Supplementary Table 1) were indicative of post-operative systemic inflammation.

Plasma levels of neutrophil-associated markers increase immediately postsurgery necessitating CPB Plasma levels of S100A8/A9 (calprotectin) were significantly ( $\mathrm{p}<0.0001)$ higher $(8.29 \mu \mathrm{g} / \mathrm{ml}$, IQR 5.96$11.68)$ immediately $(<30 \mathrm{~min})$ post-CPB, compared with pre-operative levels $(0.76 \mu \mathrm{g} / \mathrm{ml}$, IQR $0.59-0.10$, $\mathrm{n}=39$; Figure 1A). Basal levels of S100A12 were substantially lower than those of S100A8/A9, but increased significantly $(\mathrm{p}<0.0001)$ from pre-operative levels of $8.5 \mathrm{ng} / \mathrm{ml}$ (IQR 4.1-13.1) to $144.6 \mathrm{ng} / \mathrm{ml}$ (IQR 86.7-206.7), immediately postsurgery $(\mathrm{n}=38$; Figure $1 \mathrm{~B})$. Plasma levels of MPO were also significantly $(\mathrm{p}<0.0001)$ higher (198 ng/ml, IQR 149-243.5) immediately postoperatively, when compared with pre-operative levels $(6 \mathrm{ng} / \mathrm{ml}$, IQR 3-9, $\mathrm{n}=39$; Figure 1C). Plasma levels of these markers decreased to pre-operative values by $24 \mathrm{~h}$ (data not 
Table 1. Pre-operative characteristics, operative variable and post-operative outcomes of patients undergoing cardiac surgery requiring cardiopulmonary bypass. ${ }^{\dagger}$

\begin{tabular}{|c|c|}
\hline Variables & Values \\
\hline \multicolumn{2}{|l|}{ Pre-operative characteristics } \\
\hline Age, mean $(S D)$ years & $66.5(12.7)$ \\
\hline Gender F/M & $13 / 26$ \\
\hline BMI, mean (SD) kg/m² & $27.1(4.1)$ \\
\hline Creatinine, median (IQR) $\mu \mathrm{mol} / \mathrm{I}$ & $86(71-100)$ \\
\hline EuroSCORE, mean & $4.87 \pm 2.46$ \\
\hline \multicolumn{2}{|l|}{ Operative variables } \\
\hline \multicolumn{2}{|l|}{ Operation } \\
\hline AVR & 16 \\
\hline MVR & 9 \\
\hline$A V R+C A B G$ & 6 \\
\hline $\mathrm{MVR}+\mathrm{CABG}$ & 3 \\
\hline AVR + other & 2 \\
\hline MVR + other & 3 \\
\hline CPB duration, median (IQR) min & $118(94-148)$ \\
\hline Ischemic time, mean (SD) min & $91.5(32.1)$ \\
\hline \multicolumn{2}{|l|}{ Postoperative outcomes } \\
\hline $\mathrm{PaO}_{2}: \mathrm{FiO}_{2}$ ratio (POD1), mean (SD), $\mathrm{mmHg}$ & $268.3(87.7)$ \\
\hline Arteriolar-alveolar oxygen gradient, mean (SD) & $22.2(8.7)$ \\
\hline Duration of ventilation, median (IQR) $\mathrm{h}$ & $15.4(11.1-19.1)$ \\
\hline APACHE II score, mean (SD) & $6.9(3.2)$ \\
\hline ICU LOS, median (IQR) h & $23.9(20.6-47.0)$ \\
\hline Level 3 care duration, median (IQR) $\mathrm{h}$ & $16.6(11.6-20.3)$ \\
\hline Hospital LOS, median (IQR) days & $10(7-14)$ \\
\hline Incidence of AKI n (\%) & $7 / 39(18 \%)$ \\
\hline Hospital mortality, n (\%) & 0 \\
\hline \multicolumn{2}{|c|}{$\begin{array}{l}{ }^{\dagger} \mathrm{n}=39 \text { patients undergoing cardiac surgery requiring CPB. } \\
\text { AKI: Acute kidney injury; APACHE: Acute Physiology and Chronic Health Evaluation; AVR: Aortic valve repair/replacement/redo; CABG: Coronary artery bypass grafting; CPB: Cardiopul- } \\
\text { monary bypass; F: Female; ICU: Intensive care unit; IQR: Interquartile range; LOS: Length of stay; M: Male; MVR: Mitral valve repair/replacement; POD: Postoperative day; SD: Standard } \\
\text { deviation. }\end{array}$} \\
\hline
\end{tabular}

shown). Levels of cfDNA also increased significantly ( $\mathrm{p}=0.03$ ), albeit by a small amount, from $47.6 \mathrm{ng} / \mathrm{ml}$ (IQR, $41.2-54.0$ ) pre-operatively to $50.4 \mathrm{ng} / \mathrm{ml}$ (IQR, 45.7-59.3) directly after CPB ( $\mathrm{n}=38$; Figure 1D).

Associations were sought for pre- and postoperative plasma levels of S100A8/A9 with patient characteristics (as listed in Table 1 but not including age); and with markers of systemic inflammation (as in Supplementary Table 1). A number of significant, positive associations were found with postoperative plasma levels of S100A8/A9; although fewer with pre-operative levels (Table 2). Likewise, there were few significant associations either between pre- or postoperative plasma levels of S100A12, MPO and cfDNA, although associations with CPB time and PMNs immediately after $\mathrm{CPB}$ and on POD day 2, were observed for each marker (Supplementary Tables 2, $3 \& 4$ ).

Patients who developed AKI 1 week postsurgery had higher levels immediately postoperatively of S100A8/A9, S100A12 and MPO but not cfDNA.

When the patient cohort was considered as one, there was no significant difference between pre-operative creatinine levels $(86 \mu \mathrm{mol} / \mathrm{l}, \mathrm{IQR} 71-100)$ and those immediately postoperatively $(81 \mu \mathrm{mol} / \mathrm{l}$, IQR $69-102)$ or on any day up to POD7 (Supplementary Table 5). When patients were divided according to whether or not they met the criteria for AKI within 1 week following surgery; the 7 patients who developed AKI had significantly higher levels immediately postoperatively of: S100A8/A9 ( $p=0.011)$, S100A12 ( $p=0.0304)$, MPO ( $p=0.0129)$, but not of cfDNA (Figure 2A-D). Neither CRP nor IL-8 plasma levels were significantly different between \pm AKI groups (data not shown). Also, there was no significant difference between PMNs between groups, although there was a significant difference in WCC (Supplementary Figure 1A \& B). 


\section{(A)}

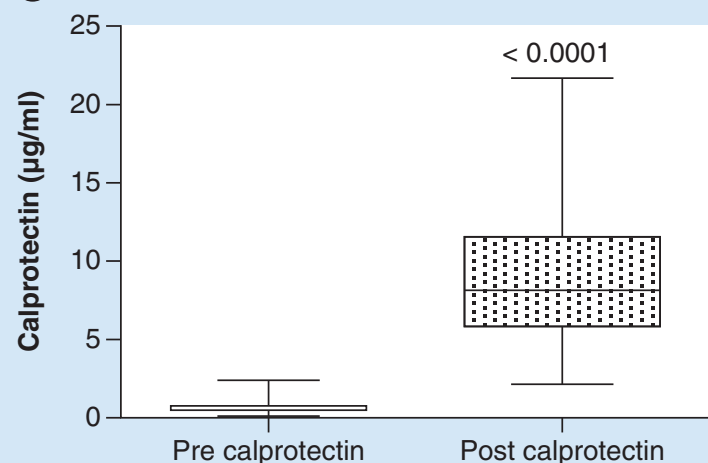

(C)

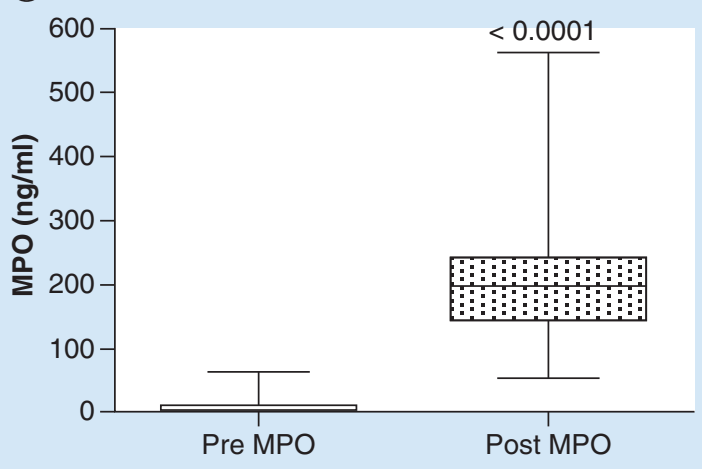

(B)

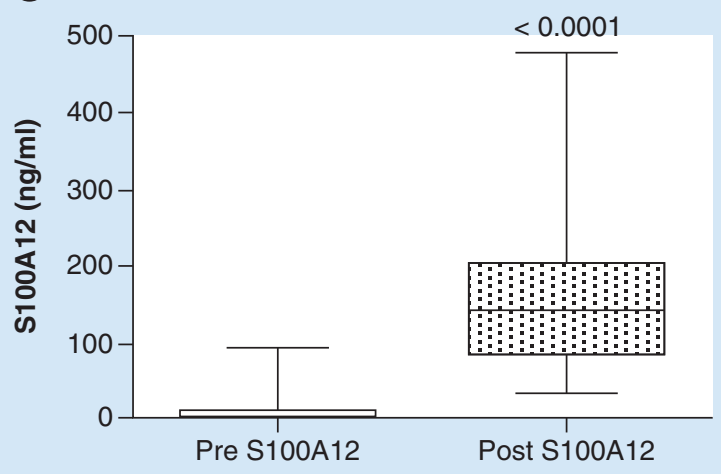

(D)

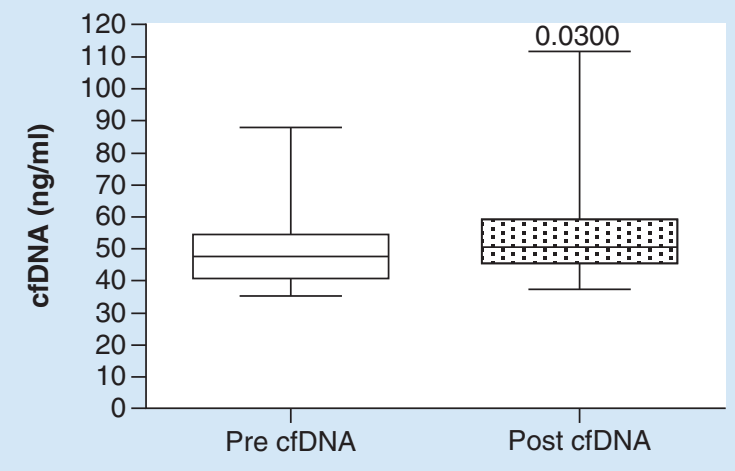

Figure 1. Plasma levels of S100A8/A9, S100A12, myeloperoxidase and cell-free DNA before and after cardiac surgery necessitating cardiopulmonary bypass. Plasma levels increased immediately ( $<30 \mathrm{~min})$ postsurgery compared with pre-operative levels. Data are presented as box and whisker plots showing median, interquartile range concentrations $(\mathrm{g} / \mathrm{ml})$ of (A) S100A8/A9 ( $\mathrm{n}=39) ;(B)$ S100A12 ( $=38) ;(C)$ MPO; $n=39 ;$ and (D) cfDNA; $n=38$. Statistical significance compared with pre-operative levels was assessed using a Wilcoxon-matched pairs test. cfDNA: Cell-free DNA.

Applying a binomial logistic model to the baseline data, we generated ROC curves to determine the predictive power of S100A8/A9, S100A12 and MPO for onset of AKI. The AUC for S100A8/A9 was 0.8125 (95\% CI: $0.676-0.950)$, with a cut-off of $10.61 \mu \mathrm{g} / \mathrm{ml}$ (85\% sensitivity, $75 \%$ specificity) (Figure 3 ) and had good predictive power. In comparison the AUCs for S100A12 and MPO were 0.7558 (95\% CI: 0.546-0.965) and 0.8065 (95\% CI: 0.653-0.960) (Supplementary Figure 2).

\section{Postoperative S100A8/A9 levels were not associated with patient age}

When patients were grouped according to age ( $<65$ or $\geq 65$ years), although there was no significant difference in WCC and PMNs immediately postoperatively, between the two age groups; by POD1, significantly higher (0.0031 and 0.002, respectively) numbers were reported in the older age group (Figure $4 \mathrm{~A} \& \mathrm{~B}$ ). There were also positive associations between age and POD1 WCC $(r=0.4108, \mathrm{p}=0.0104)$ and POD1 PMNs $(r=0.425$, $\mathrm{p}=0.0069)$. When S100A8/A9 levels were similarly grouped, there were neither significant differences between levels measured immediately postoperatively in the older compared with the younger age groups (Figure 5); nor positive associations between pre- and postoperative S100A8/A9 levels, with age $\left(\mathrm{r}^{2}=0.01198, \mathrm{p}=0.5071\right.$ and $\mathrm{r}^{2}=0.0033, \mathrm{p}=0.7287$, respectively). Likewise, postoperative levels of none of the other plasma markers measured at the time point of relevance to this study, in other words, $<30 \mathrm{~min}$ postoperatively, showed associations with age (Supplementary Table 6). By contrast, when pre-operative levels of plasma markers were considered, age was negatively associated with cfDNA levels $(-0.3853, \mathrm{p}=0.0154)$, but not MPO or S100A12 levels (Supplementary Table 6). 
(A)

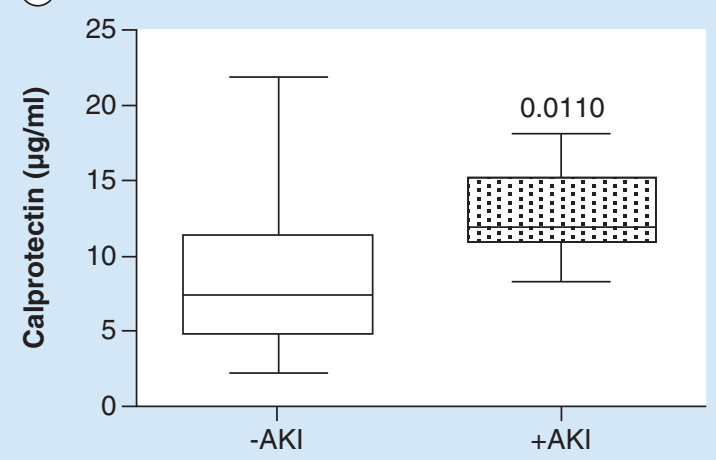

(C)

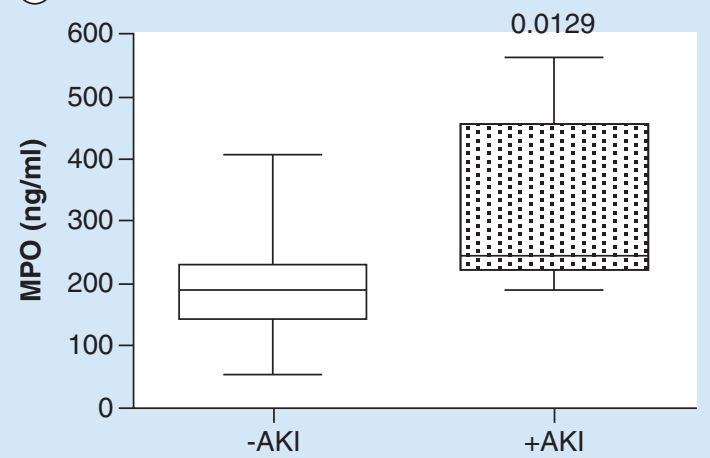

(B)

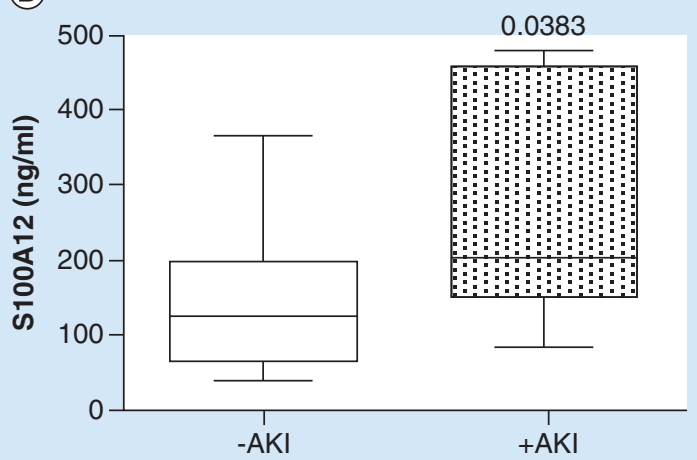

(D)

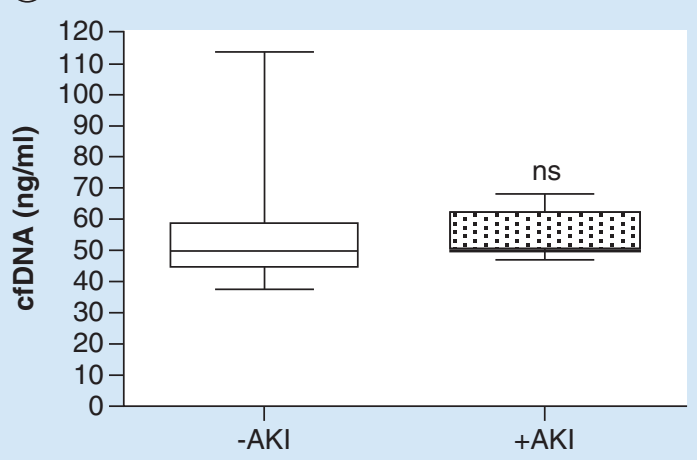

Figure 2. Plasma levels of S100A8/A9, S100A12, myeloperoxidase and cell freeDNA in patients with or without acute kidney injury. Plasma levels of S100A8/A9, S100A12 and MPO but not cfDNA immediately post-surgery ( $<30 \mathrm{~min}$ ) were higher in those patients who subsequently developed AKI ( $+\mathrm{AKI}$ ) within 7 days postsurgery than those who did not (-AKI), as assigned according to AKIN clinical classification. Data are presented as median, interquartile range concentrations $(\mu \mathrm{g} / \mathrm{ml})$ of $(A)$ S100A8/A9 and (D) cfDNA $(n=32,-A K I$ and $n=7,+A K I)$ or $(B)$ S100A12 and (C) MPO ( $n=31,-A K I$ and $n=7,+A K I)$; with significance determined using a Mann-Whitney U-test. AKI: Acute kidney injury; cfDNA: Cell-free DNA; ns: Not statistically significant.

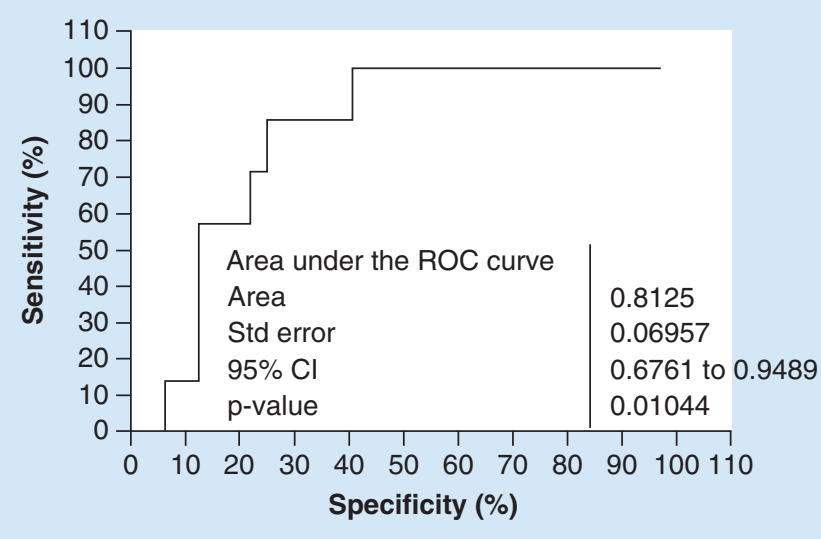

Figure 3. Receiver operating characteristic curve for S100A8/A9. The sensitivity, specificity and area under curve were calculated using a conventional ROC curve. Area under curve of 0.8125 ( $95 \% \mathrm{Cl}: 0.676-0.950)$ supports the utility of S100A8/A9 as a good biomarker for acute kidney injury. The cut-off value determined using a sensitivity of $85 \%$ sensitivity and a specificity of $75 \%$, was $10.61 \mu \mathrm{g} / \mathrm{ml}$.

$\mathrm{Cl}$ : Confidence interval; ROC: Receiver operating characteristic; Std error: Standardard error 
Table 2. Significant associations between pre- and postoperative levels of S100A8/A9 with: pre-operative characteristics, operative variables, postoperative outcomes, indices of systemic inflammation and other neutrophil-derived markers.

\begin{tabular}{|c|c|c|}
\hline Variables & Pre-operative S100A8/A9 levels & Postoperative S100A8/A9 levels \\
\hline \multicolumn{3}{|c|}{ Pre-operative characteristics } \\
\hline Pre-op S100A12 & $r=0.335, p=0.0396$ & \\
\hline Pre-op MPO & $r=0.6204, p<0.001$ & \\
\hline \multicolumn{3}{|l|}{ Operative variables } \\
\hline CPB duration & & $r=0.6276, p<0.0001$ \\
\hline Ischemic time & & $r^{2}=0.2362, p=0.0017$ \\
\hline \multicolumn{3}{|l|}{ Postoperative outcomes } \\
\hline Duration of ventilation & & $r=0.3275, p=0.3275$ \\
\hline Level 3 care & & $r=0.5196, p=0.0007$ \\
\hline Creatinine, POD2 & & $r=0.3349, p=0.0372$ \\
\hline \multicolumn{3}{|l|}{ Systemic inflammation } \\
\hline WCC post-op $<30$ min & & $r^{2}=0.2363, p=0.0020$ \\
\hline WCC POD1 & & $r=0.3484, p=0.0320$ \\
\hline WCC POD2 & & $r=0.4962, p=0.0015$ \\
\hline PMNs post-op < $30 \mathrm{~min}$ & & $r=0.5654, p=0.0002$ \\
\hline PMNs POD2 & & $r=0.4968, p=0.0013$ \\
\hline \multicolumn{3}{|c|}{ Other neutrophil-associated markers } \\
\hline S100A12 & & $r=0.7068, p<0.0001$ \\
\hline MPO & & $r=0.5578, p=0.0003$ \\
\hline cfDNA & & $r=0.4972, p=0.0013$ \\
\hline
\end{tabular}

cfDNA: Cell-free DNA; CPB: Cardiopulmonary bypass; PMN: Neutrophil number; POD: Post-operative day; Pre-op: Pre-operative; r: Spearman's correlation; $r^{2}$ : Pearson's correlation, with associated significance $(p)$ value; WCC: White cell count.

(A)

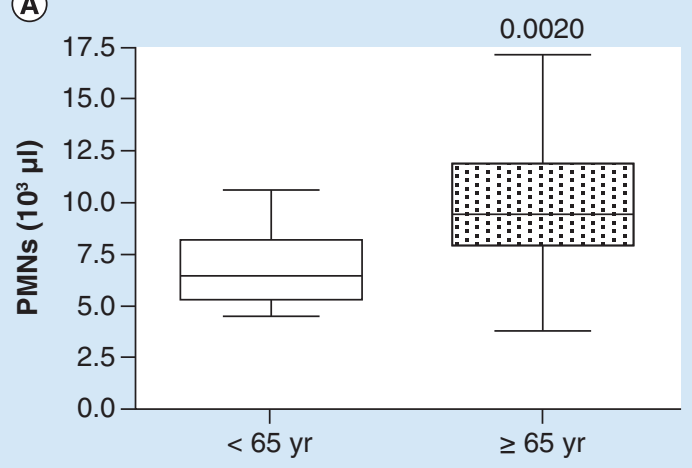

(B)

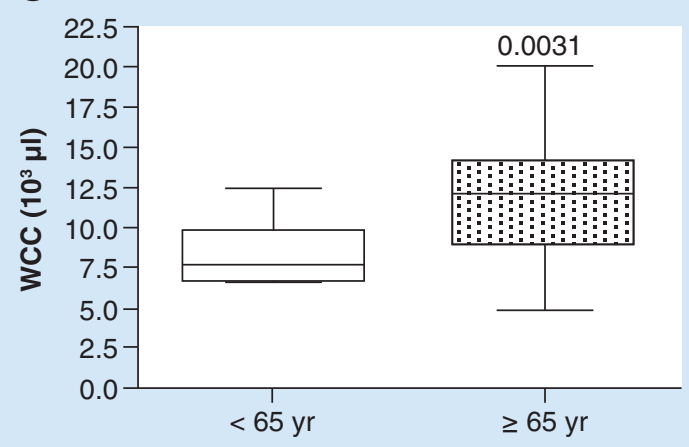

Figure 4. Neutrophil and white cell counts in patients grouped according to age. PMNs and WCC were significantly higher in patients aged 65 years and above compared with those of less than 65 years. Data are presented as median, interquartile range $\left(10^{3} \mu\right.$ l) of (A) PMNs $(n=17,<65$ years; and $n=22$ years, $\geq 65)$; and (B) WCC $(n=16,<65$ years; and $\mathrm{n}=22$ years, $\geq 65$ ) on postoperative day 1 ; with significance determined using a Mann-Whitney U-test.

PMN: Neutrophil numbers; WCC: White cell count.

\section{Discussion}

This exploratory study showed that plasma levels of S100A8/A9 increased immediately postoperatively in patients who had undergone surgery necessitating CPB. Likewise, levels of S100A12, MPO and cfDNA also increased. Postoperative levels of S100A8/A9 correlated with length of CPB and ischemic time; and also duration of ventilation and requirement for level 3 care. Fewer and weaker associations were found with S100A12, MPO and cfDNA. When patients were grouped according to whether or not AKI developed within 7 days postsurgery, significantly higher levels of S100A8/A9, S100A12 and MPO, but not cfDNA, were detected in the AKI group; and of these, 


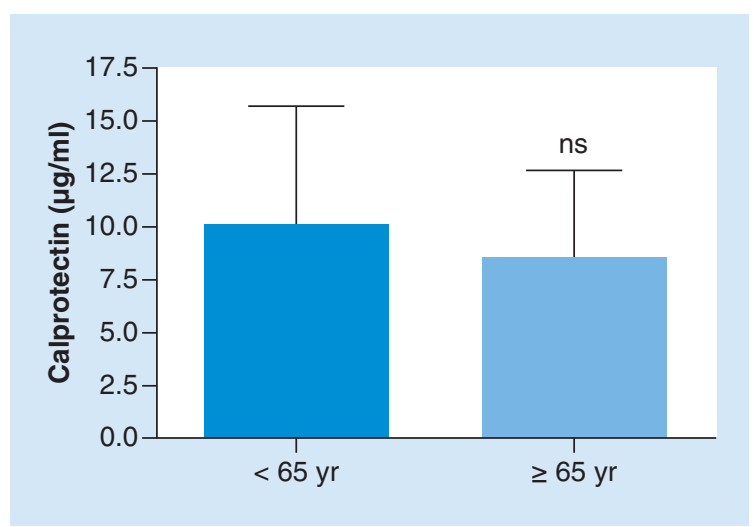

Figure 5. S100A8/A9 levels grouped according to age. Postoperative plasma levels of S100A8/A9 were not significantly different in patients aged 65 years and above compared with those of less than 65 years. Data are presented as median, interquartile range concentrations $(\mu \mathrm{g} / \mathrm{ml})$ of S100A8/A9; with significance determined using a Mann-Whitney U-test. ns: Not statistically significant.

S100A8/A9 had strong good prognostic value for predicting AKI onset. Moreover, S100A8/A9 levels were not altered with age. These novel findings suggest that S100A8/A9 levels, measured immediately postoperatively, could be a useful, early indicator of the likelihood of developing CSA-AKI, irrespective of age.

While the number of patients we studied was relatively small, this current study was a logical progression of a much larger study already published by our group; a study specifically designed to establish the epidemiology of AKI after cardiac surgery [2]. In this retrospective analysis of nearly 1900 patients we evaluated the utility of each of the Risk/Injury/Failure/Loss/End-stage (RIFLE) criteria, AKIN classification (modified RIFLE) and the Kidney Disease: Improving Global Outcomes (KDIGO) classification for AKI postcardiac surgery. In this larger cohort study, the incidence of AKI using the AKI and RIFLE criteria were 25.9 and 24.9\%, respectively, but individual patients were classified differently. Incidence and outcome according to AKIN and KDIGO classification were identical. The percentage receiving renal replacement therapy was $6.2 \%$ and associated hospital mortality was $15.6 \%$. Reported incidences of CSA-AKI in other studies vary from 17 to $30 \%$. Indeed, we have also previously shown in another small patient cohort an incidence of $17.9 \%$ [4].

S100A8/A9 constitutes $40 \%$ of the cytosolic protein in neutrophils and is expressed at lower levels (5\%) in monocytes [8]. It is released either through nonclassical secretion, or loss of cell membrane integrity during necrosis and acts as a neutrophil chemoattractant and activator. Our findings of increased levels of S100A8/A9 in patients undergoing cardiac surgery support previous reports of increased expression in acute and chronic inflammatory conditions, including sepsis and septic shock [41,42]. Plasma S100A8/A9 levels in healthy controls reported in the sepsis study were similar to our pre-operative levels; and our postoperative levels were in a similar range to those in patients with septic shock [42]. Our current findings also extend our previous observations in a separate patient cohort showing raised S100A8/A9 at $2 \mathrm{~h}$ postsurgery [15]. Also, our findings of increased plasma S100A8/A9 post$\mathrm{CPB}$ corroborate a study by another group showing greater mRNA expression of S100A9 in circulating neutrophils following CPB [16]. Expectedly, plasma S100A8/A9 levels correlated with PMNs, WCC and also S100A12 and MPO levels. Moreover, S100A8/A9 and cfDNA levels were also positively associated. In response to infection, S100A8/A9 is released from neutrophils as a NETs-associated protein [8] and might also be released, similarly, during $\mathrm{CPB}$ surgery; providing a rationale for our association with cfDNA.

In terms of correlations between S100A8/A9 levels and operative parameters, there was, as we have previously shown, a positive association with duration of $\mathrm{CPB}$ and also in this study, with ischemic time. These associations suggest that injurious stimuli resulting from surgery/CPB including: tissue hypoperfusion, operative tissue injury, myocardial and pulmonary ischemia; and activation of blood during exposure to extracorporeal circuits [43] are likely to contribute to release of S100A8/A9. In our previous study S100A8/A9, at $2 \mathrm{~h}$, did not correlate with outcome parameters [15]. By contrast, in the current study, plasma concentrations immediately after surgery, were significantly associated with duration of ventilation and level 3 care. Thus, it is likely that these earlier measurements better reflect release consequent on the operative procedure, and thus, could be a useful indicator of recovery, which that can be measured immediately, postoperatively.

S100A12 is also an abundant neutrophil-calgranulin protein, but unlike S100A8/A9 its expression is almost completely restricted to neutrophils [19,44]. In support of this, we showed that plasma S100A8/A9 levels associated with postoperative PMNs only and not with WCC. A previous study in 25 adult patients undergoing coronary artery bypass grafting also showed raised S100A12 levels at $4 \mathrm{~h}$ postsurgery, correlated with duration of CPB [20]. 
A further study, in children undergoing cardiac surgery requiring CPB, showed S100A12 increased in plasma postoperatively and that levels correlated with respiratory parameters, ICU and hospital LOS [21]. By contrast, raised levels in our study correlated with duration of level 3 care only, and not specific respiratory parameters, possibly because, unlike the children in the study by Liu et al., none of our adult patients developed acute lung injury [21].

MPO has long been considered as a marker of primary neutrophil granules and release in response to stimuli associated with cardiac surgery, suggests profound neutrophil activation in this setting. The tenfold increase seen in our study is of a similar order of magnitude to postsurgery increases reported in other studies, and indeed, as in our study, correlation with length of CPB time has been noted by others [45]. However, what is perhaps surprising is that, MPO levels did not correlate with either postsurgery plasma IL-8 levels, as others have shown, or indeed with levels of either calgranulin. One explanation might be the early time point at which our measurements were made; highlighting the value of mediators that can be measured immediately after surgery and have diagnostic/prognostic value such as S100A8/A9.

Circulating cfDNA, extracellular DNA found in blood or plasma, has been widely considered as a potential marker for the detection and monitoring of a wide range of human diseases. Our negative correlation between pre-operative cfDNA levels and age is of note. A previous study in patients with rheumatoid arthritis (RA) showed lower levels in patients with established (es) versus early (e)RA; the median age of patients with esRA was greater than the eRA group, although speculation about a possible effect of age on cfDNA was not made [46]. Generally, cfDNA levels increase with disease, with highest levels indicative of worse outcome, as seen in patients with severe sepsis $[25,29]$. Moreover, it has been suggested that plasma cfDNA levels are a better indicator of ICU mortality than multiple organ dysfunction or APACHE II scores [29]. However, levels reported in sepsis patients are very much higher than those reported in our study [25], most likely because within 30 min postsurgery is a very early time, in terms of systemic inflammation; future studies at later time points are warranted.

Perhaps also because of the early time point selected, cfDNA was the one plasma marker that did not show a significant difference, in this study, between those patients who went on to manifest AKI within a week of surgery and those who did not. By contrast, the calgranulins and MPO showed significant difference between the two groups. The differences between S100A8/A9 levels were the most significant and gave rise to highest area under the ROC curve for predicting AKI onset. Indeed, our value of 0.81 from AUC for S100A8/A9 is similar to that shown by Kashani et al. who showed in a larger, multicenter study that a combination of urine levels of TIMP-2 and IGFBP-7 gave similar predictive values for AKI [47].

The need to predict and prevent AKI has become possibly the most pressing clinical problem to be anticipated and addressed in patients undergoing cardiac surgery. As we and others have shown, AKI has adverse prognostic significance [2,3]. Moreover, the now well-recognised link between AKI and the onset of chronic kidney disease [48] would also suggest that finding plasma markers that might identify those patients most at risk of developing AKI - and then protecting them accordingly - could have far reaching consequences. Therefore, there is an increasing interest in the development of early AKI biomarkers to enable intervention, as it is a severe complication which increases the likelihood of mortality. Other new potential biomarkers for AKI, detected in blood serum or urine, have been proposed in the last few years, such as NGAL, Cys-C, KIM-1, FABPs, IL-18, EO, TIMP-2 and IGFBP7 [7,49]. While we acknowledge that our study on the S100A8/A9 as a potential biomarker for AKI is restricted to patients developing AKI post cardiac surgery, other studies have also proposed a link between calprotectin and AKI and its potential use as a valuable biomarker [49]. It was shown that raised levels of urinary S100A8/A9 can predict the likelihood of rejection after renal transplant [50] and can also distinguish between prerenal and intrinsic acute kidney or allograft injury [51-55]. Serum S100A8/A9 positive leukocytes are also associated with glomerular inflammation in ANCA-associated vasculitis and glomerulonephritis [56]. Also, plasma levels of S100A8/A9 proved to be a useful marker of inflammation in chronically dialysed patients with end stage renal disease [57]. Interestingly, a recent study actually showed that the S100A8/A9-TLR4-NLRP3 inflammasome pathway is a key mechanism of contrast-induced AKI (CI-AKI), as the levels of S100A8/A9 but also TLR4 and NLRP3 increased in in vivo and in vitro models of AKI [58]. Based on these findings together with our own, we suggest that S100A8/A9 is a very promising candidate molecule that merits further investigation as a marker of CSA-AKI.

In the elderly, AKI is associated with considerable mortality [6]. Moreover, the age of patients undergoing cardiac surgery is increasing, with a 24 -fold increase in patients $>80$ years old since 1990 s [59]. When selecting inflammatory biomarkers for diagnostic and prognostic use, particularly in an ageing patient population, it is important that expression is independent of age. In general, the immune system becomes less efficient with age, a phenomenon 
referred to as immunosenescence [34]. However, ageing is also, paradoxically, associated with a prolonged and upregulated inflammatory response, known as inflammaging [33]. To what extent these paradigms might impact on inflammatory markers selected to predict surgical outcome, is not known. We showed that WCC and PMNs increased with age in our patient group. This could in part be explained by the observation that with age, generally more myeloid than lymphoid progenitors are produced from hematopoietic stem cells, ensuring PMNs are at least maintained [60]; or as is the case in our patient group, increased. However, there are a number of conflicting studies as to the manner in which circulating neutrophil levels change in the elderly [61] and it might well be context dependent. Few studies have investigated the perioperative release of inflammatory mediators at different ages. One such study showed similar cytokine release in an older versus a younger age group [62]; similar to our finding with S100A8/A9 and also S100A12 and MPO. A study in elderly patients with chronic rhinosinusitis showed age-related decrease in S100A8/A9 expression in nasal lavage and polyp tissue [63,64]. By contrast, a study that investigated S100A8 expression in ageing skin showed increased expression with age [65]. However, as with PMN these findings are likely to be context dependent, especially in these chronic, as opposed to acute scenarios as seen in our study.

We acknowledge limitations to our investigation including a lack of standardisation of peri-operative care. Also, there was no standard protocol for management by the anesthetists, perfusionists or cardiac surgeons; which might have varied slightly between patients, but this is a common problem with many similar observational cohort studies. Further, the inflammatory response and hence S100A8/A9 levels could be influenced by the anesthetic and neuromuscular blocking agents employed and also the fluid resuscitation strategy. Another limitation could be the lack of information about all pre-operative medications which again might influence S100A8/A9 levels. Also, level 3 dependency is not a perfect surrogate for critical illness, but is potentially more sensitive than the length of time that a patient is located within an ICU. Finally, the size of the cohort is small but the incidence of AKI falls within those previously reported.

In conclusion, while the exact nature of the role of S100A8/A9 in AKI following cardiac surgery with CPB cannot be concluded in this hypothesis-driven study, further research is merited to elucidate its role. Such ongoing studies will contribute insight to the ongoing debate on the use of anti-inflammatory strategies to treat AKI [66] and lead to the development of possible novel therapeutic strategies. While we acknowledge that a number of biomarkers have recently been suggested for AKI, our study proposing early measurement of plasma S100A8/A9, further helps to bridge the gap associated with creatinine measurements alone as indicators of AKI. In the interim, we suggest that measuring plasma S100A8/A9 levels immediately post-CPB, alone or in combination with other markers, is a very valuable indicator of the likelihood of CSA-AKI onset.

Supplementary data

To view the supplementary data that accompany this paper please visit the journal website at: www.futuremedicine.com/doi/full/10.2217/bmm-2018-0238

\section{Acknowledgments}

The authors thank V Pingel, Royal Brompton Hospital for assistance in collecting clinical data and M Smith, Imperial College London, School of Medicine, for the measurement of S100A12. The authors would also like to thank the Dunhill Medical Trust for funding Z Nikolakopoulou.

Financial \& competing interests disclosure

The authors have no relevant affiliations or financial involvement with any organization or entity with a financial interest in or financial conflict with the subject matter or materials discussed in the manuscript. This includes employment, consultancies, honoraria, stock ownership or options, expert testimony, grants or patents received or pending, or royalties. No writing assistance was utilized in the production of this manuscript.

Ethical conduct

The research was carried out in accordance with the Declaration of Helsinki (2013) of the World Medical Association and the study protocol was approved by the Research Ethics Committee for the Royal Brompton and Harefield NHS Foundation Trust. Informed, written consent was obtained (one day before surgery and after determining patient suitability) from all patients. Reporting of the study conforms to STROBE and broader EQUATOR guidelines [38]. 


\section{Summary points}

- There is an increased need in the identification of new predictive biomarkers of acute kidney injury (AKI) in the early stages to enable therapeutic intervention.

- $\mathrm{AKI}$ is one of the complication and pressing clinical problems of patients undergoing cardiac surgery and can increase the likelihood of mortality.

Method

- This exploratory study involved plasma samples of 39 patients undergoing cardiac surgery necessitating cardiopulmonary bypass (CPB) before and immediately post-CPB.

- It was investigated whether early neutrophils-derived plasma markers, particularly S100A8/A9 can predict AKI onset in these patients.

- All the markers investigated increased significantly immediately post-CPB.

Results

- Postoperative S100A8/A9 levels were significantly associated with perioperative characteristics including: cell numbers, duration or CPB and outcome variables.

- S100A8/A9, S100A12 and MPO levels were all significantly higher, immediately post-CPB, in those patients who developed AKI within 7 days of surgery.

- S100A8/A9 was the most promising of all the markers measured, showing a very good prognostic utility for AKI, with an area under the receiver operating characteristic curve of 0.81 and a cut-off value of $10.6 \mu \mathrm{g} / \mathrm{ml}$ irrespective of age.

Conclusion

- Measurement of S100A8/A9 immediately post-CPB, alone or in combination with other markers, can be a valuable indicator of AKI onset after cardiac surgery.

\section{Open access}

This work is licensed under the Attribution-NonCommercial-NoDerivatives 4.0 Unported License. To view a copy of this license, visit http://creativecommons.org/licenses/by-nc-nd/4.0/

\section{References}

Papers of special note have been highlighted as: • of interest; $\bullet \bullet$ of considerable interest

1 MacCallum NS, Finney SJ, Gordon SE, Quinlan GJ, Evans TW. Modified criteria for the systemic inflammatory response syndrome improves their utility following cardiac surgery. Chest 145(6), 1197-1203 (2014).

2 Bastin AJ, Ostermann M, Slack AJ, Diller GP, Finney SJ, Evans TW. Acute kidney injury after cardiac surgery according to Risk/Injury/Failure/Loss/End-stage, Acute Kidney Injury Network, and Kidney Disease: improving global outcomes classifications. J. Crit. Care 28(4), 389-396 (2013).

-• A retrospective analysis of nearly 1900 patients to establish the epidemiology of acute kidney injury (AKI) after cardiac surgery.

3 Mao H, Katz N, Ariyanon W et al. Cardiac surgery-associated acute kidney injury. Cardiorenal Med. 3(3), 178-199 (2013).

4 Burke-Gaffney A, Svermova T, Mumby S, Finney SJ, Evans TW. Raised plasma robo4 and cardiac surgery-associated acute kidney injury. PLoS ONE 9(10), e111459 (2014).

5 Rinder CS, Fontes M, Mathew JP, Rinder HM, Smith BR. Neutrophil CD11b upregulation during cardiopulmonary bypass is associated with postoperative renal injury. Ann. Thorac. Surg. 75(3), 899-905 (2003).

6 Gong Y, Zhang F, Ding F, Gu Y. Elderly patients with acute kidney injury (AKI): clinical features and risk factors for mortality. Arch. Gerontol. Geriatr. 54(2), e47-e51 (2012).

7 Pozzoli S, Simonini M, Manunta P. Predicting acute kidney injury: current status and future challenges. J. Nephrol. 31(2), 209-223 (2018).

8 Vogl T, Gharibyan AL, Morozova-Roche LA. Pro-inflammatory S100A8 and S100A9 proteins: self-assembly into multifunctional native and amyloid complexes. Int. J. Mol. Sci. 13(3), 2893-2917 (2012).

9 Bertheloot D, Latz E. HMGB1, IL-1alpha, IL-33 and S100 proteins: dual-function alarmins. Cell. Mol. Immunol. 14(1), 43-64 (2016).

10 Pruenster M, Vogl T, Roth J, Sperandio M. S100A8/A9: from basic science to clinical application. Pharmacol. Ther.167, 120-131 (2016).

11 Ryckman C, Vandal K, Rouleau P, Talbot M, Tessier PA. Proinflammatory activities of S100: proteins S100A8, S100A9, and S100A8/A9 induce neutrophil chemotaxis and adhesion. J. Immunol. 170(6), 3233-3242 (2003).

- This study showed the important pro-inflammatory role of S100A8/A9 inducing neutrophil chemotaxis and adhesion. 
12 Sunahori K, Yamamura M, Yamana J et al. The S100A8/A9 heterodimer amplifies proinflammatory cytokine production by macrophages via activation of nuclear factor kappa B and p38 mitogen-activated protein kinase in rheumatoid arthritis. Arthritis Res. Ther. 8(3), R69 (2006).

13 Doussiere J, Bouzidi F, Vignais PV. A phenylarsine oxide-binding protein of neutrophil cytosol, which belongs to the S100 family, potentiates NADPH oxidase activation. Biochem. Biophys. Res. Commun. 285(5), 1317-1320 (2001).

14 Ikemoto M, Matsumoto S, Egawa $\mathrm{H}$ et al. A case with transient increases in serum S100A8/A9 levels implying acute inflammatory responses after pancreatic islet transplantation. Ann. Clin. Biochem. 44(Pt 6), 570-572 (2007).

15 Creagh-Brown BC, Quinlan GJ, Hector LR, Evans TW, Burke-Gaffney A. Association between preoperative plasma sRAGE levels and recovery from cardiac surgery. Mediators Inflamm. 2013, 496031 (2013).

-. This study showed that plasma levels of S100A8/A9 increased $2 \mathrm{~h}$ postsurgery in patients undergoing surgery necessitating cardiopulmonary bypass.

16 Pavelkova M, Kubala L, Ciz M et al. Blood phagocyte activation during open heart surgery with cardiopulmonary bypass. Physiol. Res. 55(2), 165-173 (2006).

17 Robinson MJ, Hogg N. A comparison of human S100A12 with MRP-14 (S100A9). Biochem. Biophys. Res. Commun. 275(3), 865-870 (2000).

18 Miranda LP, Tao T, Jones A et al. Total chemical synthesis and chemotactic activity of human S100A12 (EN-RAGE). FEBS Lett. 488(1-2), 85-90 (2001).

19 Vogl T, Propper C, Hartmann M et al. S100A12 is expressed exclusively by granulocytes and acts independently from MRP8 and MRP14. J. Biol. Chem. 274(36), 25291-25296 (1999).

20 Scheiber-Camoretti R, Mehrotra A, Yan L, Raman J, Beshai JF, Hofmann Bowman MA. Elevated S100A12 and sRAGE are associated with increased length of hospitalization after non-urgent coronary artery bypass grafting surgery. Am. J. Cardiovasc. Dis. 3(2), 85-90 (2013).

21 Liu X, Chen Q, Shi S et al. Plasma sRAGE enables prediction of acute lung injury after cardiac surgery in children. Crit. Care 16(3), R91 (2012).

22 Larson DF, Bowers M, Schechner HW. Neutrophil activation during cardiopulmonary bypass in paediatric and adult patients. Perfusion 11(1), 21-27 (1996).

23 Kahli A, Guenancia C, Zeller M et al. Growth differentiation factor-15 (GDF-15) levels are associated with cardiac and renal injury in patients undergoing coronary artery bypass grafting with cardiopulmonary bypass. PLoS ONE 9(8), e105759 (2014).

24 Brinkmann V, Reichard U, Goosmann C et al. Neutrophil extracellular traps kill bacteria. Science 303(5663), 1532-1535 (2004).

25 Avriel A, Paryente Wiessman M, Almog Y et al. Admission cell free DNA levels predict 28-day mortality in patients with severe sepsis in intensive care. PLoS ONE 9(6), e100514 (2014).

26 Saukkonen K, Lakkisto P, Varpula M et al. Association of cell-free plasma DNA with hospital mortality and organ dysfunction in intensive care unit patients. Intensive Care Med. 33(9), 1624-1627 (2007).

27 Lam NY, Rainer TH, Chan LY, Joynt GM, Lo YM. Time course of early and late changes in plasma DNA in trauma patients. Clin. Chem. 49(8), 1286-1291 (2003).

28 Ren B, Liu F, Xu F, He J, Zhu H, Zou G. Is plasma cell-free DNA really a useful marker for diagnosis and treatment of trauma patients? Clin. Chim. Acta 424, 109-113 (2013).

29 Dwivedi DJ, Toltl LJ, Swystun LL et al. Prognostic utility and characterization of cell-free DNA in patients with severe sepsis. Crit. Care 16(4), R151 (2012).

30 Hashiba M, Huq A, Tomino A et al. Neutrophil extracellular traps in patients with sepsis. J. Surg. Res. 194(1), 248-254 (2014).

31 Rhodes A, Cecconi M. Cell-free DNA and outcome in sepsis. Crit. Care 16(6), 170 (2012).

32 Desai A, Grolleau-Julius A, Yung R. Leukocyte function in the aging immune system. J. Leukoc. Biol. 87(6), 1001-1009 (2010).

33 Franceschi C, Campisi J. Chronic inflammation (inflammaging) and its potential contribution to age-associated diseases. J. Gerontol. A Biol. Sci. Med. Sci. 69(Suppl 1), S4-S9 (2014).

34 Panda A, Arjona A, Sapey E et al. Human innate immunosenescence: causes and consequences for immunity in old age. Trends Immunol. 30(7), 325-333 (2009).

35 Shaw AC, Joshi S, Greenwood H, Panda A, Lord JM. Aging of the innate immune system. Curr. Opin. Immunol. 22(4), 507-513 (2010).

36 Qian F, Wang X, Zhang L et al. Age-associated elevation in TLR5 leads to increased inflammatory responses in the elderly. Aging Cell 11(1), 104-110 (2012).

37 Eddleston J, Goldhill D, Morris J. Levels of critical care for adult patients. Intensive Care Soci Stand. (2009). www.ics.ac.uk/professional/standards_safety_quality/standards_and_guidelines/levels_of_critical_care_for_adult_patients

38 Simera I, Moher D, Hoey J, Schulz KF, Altman DG. A catalogue of reporting guidelines for health research. Eur. J. Clin. Invest. 40(1), $35-53$ (2010). 
39 Bridgewater B, Keogh B, Kinsman R, Walton P. Sixth National Cardiac Database Report. Cardiac Surg. (2009). www.e-dendrite.com/files/13/file/Pages\%20from\%20NACSD\%202008.pdf

40 Dieleman JM, Nierich AP, Rosseel PM et al. Intraoperative high-dose dexamethasone for cardiac surgery: a randomized controlled trial. JAMA 308(17), 1761-1767 (2012).

41 Fontaine M, Pachot A, Larue A et al. Delayed increase of S100A9 messenger RNA predicts hospital-acquired infection after septic shock. Crit. Care Med. 39(12), 2684-2690 (2011).

42 Van Zoelen MA, Vogl T, Foell D et al. Expression and role of myeloid-related protein-14 in clinical and experimental sepsis. Am. J. Respir. Crit. Care Med. 180(11), 1098-1106 (2009).

43 Levy JH, Tanaka KA. Inflammatory response to cardiopulmonary bypass. Ann. Thorac. Surg. 75(2), S715-S720 (2003).

44 Guignard F, Mauel J, Markert M. Identification and characterization of a novel human neutrophil protein related to the S100 family. Biochem. J. 309(Pt 2), 395-401 (1995).

45 Gessler P, Pretre R, Hohl V, Rousson V, Fischer J, Dahinden C. CXC-chemokine stimulation of neutrophils correlates with plasma levels of myeloperoxidase and lactoferrin and contributes to clinical outcome after pediatric cardiac surgery. Shock 22(6), 513-520 (2004).

46 Dunaeva M, Buddingh BC, Toes RE, Luime JJ, Lubberts E, Pruijn GJ. Decreased serum cell-free DNA levels in rheumatoid arthritis. Auto Immun. Highlights 6(1-2), 23-30 (2015).

47 Kashani K, Al-Khafaji A, Ardiles T et al. Discovery and validation of cell cycle arrest biomarkers in human acute kidney injury. Crit. Care 17(1), R25 (2013).

48 Chawla LS, Eggers PW, Star RA, Kimmel PL. Acute kidney injury and chronic kidney disease as interconnected syndromes. N. Engl. J. Med. 371(1), 58-66 (2014).

49 Schrezenmeier EV, Barasch J, Budde K, Westhoff T, Schmidt-Ott KM. Biomarkers in acute kidney injury - pathophysiological basis and clinical performance. Acta Physiol. 219(3), 554-572 (2017).

-• A review about the pathophysiology and the most important currently proposed biomarkers of AKI.

50 Tepel M, Borst C, Bistrup C et al. Urinary calprotectin and posttransplant renal allograft injury. PLoS ONE 9(11), e113006 (2014).

51 Heller F, Frischmann S, Grunbaum M, Zidek W, Westhoff TH. Urinary calprotectin and the distinction between prerenal and intrinsic acute kidney injury. Clin. J. Am. Soc. Nephrol. 6(10), 2347-2355 (2011).

52 Chang $\mathrm{CH}$, Yang $\mathrm{CH}$, Yang HY et al. Urinary biomarkers improve the diagnosis of intrinsic acute kidney injury in coronary care units. Medicine 94(40), e1703 (2015).

53 Seibert FS, Rosenberger C, Mathia S et al. Urinary calprotectin differentiates between prerenal and intrinsic acute renal allograft failure. Transplantation 101(2), 387-394 (2017).

54 Seibert FS, Pagonas N, Arndt R et al. Calprotectin and neutrophil gelatinase-associated lipocalin in the differentiation of pre-renal and intrinsic acute kidney injury. Acta Physiol. 207(4), 700-708 (2013).

55 Kim AJ, Ro H, Kim H et al. Klotho and S100A8/A9 as discriminative markers between pre-renal and intrinsic acute kidney injury. PLoS ONE 11(1), e0147255 (2016).

56 Pepper RJ, Hamour S, Chavele KM et al. Leukocyte and serum S100A8/S100A9 expression reflects disease activity in ANCA-associated vasculitis and glomerulonephritis. Kidney Int. 83(6), 1150-1158 (2013).

57 Malickova K, Brodska H, Lachmanova J et al. Plasma calprotectin in chronically dialyzed end-stage renal disease patients. Inflamm. Res. 59(4), 299-305 (2010).

58 Tan X, Zheng X, Huang Z, Lin J, Xie C, Lin Y. Involvement of S100A8/A9-TLR4-NLRP3 inflammasome pathway in contrast-induced acute kidney injury. Cell Physiol. Biochem. 43(1), 209-222 (2017).

•• This study shows the important role of S100A8/A9 in contrast-induced AKI (CI-AKI) using in vivo and in vitro models.

59 Friedrich I, Simm A, Kötting J, Thölen F, Fischer B, Silber RE. Cardiac surgery in the elderly patient. Dtsch. Arztebl. Int. 106(25), 416-422 (2009).

60 Beerman I, Bhattacharya D, Zandi S et al. Functionally distinct hematopoietic stem cells modulate hematopoietic lineage potential during aging by a mechanism of clonal expansion. Proc. Natl Acad. Sci. USA 107(12), 5465-5470 (2010).

61 Wessels I, Jansen J, Rink L, Uciechowski P. Immunosenescence of polymorphonuclear neutrophils. Scientific WorldJournal 10, 145-160 (2010).

62 Roth-Isigkeit A, Schwarzenberger J, V Borstel T et al. Perioperative cytokine release during coronary artery bypass grafting in patients of different ages. Clin. Exp. Immunol. 114(1), 26-32 (1998).

63 Cho SH, Hong SJ, Han B et al. Age-related differences in the pathogenesis of chronic rhinosinusitis. J. Allergy Clin. Immunol. 129(3), 858.e852-860.e852 (2012).

64 Cho SH, Kim DW, Lee SH et al. Age-related increased prevalence of asthma and nasal polyps in chronic rhinosinusitis and its association with altered IL-6 trans-signaling. Am. J. Respir. Cell Mol. Biol.53(5), 601-6 (2015). 
Research Article Nikolakopoulou, Hector, Creagh-Brown, Evans, Quinlan \& Burke-Gaffney

65 Lee YM, Kim YK, Eun HC, Chung JH. Changes in S100A8 expression in UV-irradiated and aged human skin in vivo. Arch. Dermatol. Res. 301(7), 523-529 (2009).

66 Scrascia G, Guida P, Rotunno C, De Luca Tupputi Schinosa L, Paparella D. Anti-inflammatory strategies to reduce acute kidney injury in cardiac surgery patients: a meta-analysis of randomized controlled trials. Artif. Organs 38(2), 101-112 (2014). 